\title{
BUCHBESPRECHUNGEN
}

\author{
Vera Gowlland-Debbas (Hrsg.)
}

\section{United Nations Sanctions and International Law}

The Graduate Institute of International Studies, vol. 1

Kluwer Law International, The Hague/London/Boston, 2001, XIV/ 408 S., € 136,00

Dieser Band versammelt die englisch-, zum Teil auch französischsprachigen Beiträge, die auf einem Kolloquium im Juni 1999 in Genf vorgetragen worden sind. Sie sind durchweg relativ kurz gehalten und ohne allzu aufwendigen Fußnotenapparat geschrieben. Das hat den Nachteil, dass man keine geschliffenen Theoriegebäude zu lesen bekommt, dafür aber atmen die Referate die Frische der Tagungsatmosphäre. Die Herausgeberin, am berühmten Genfer Hochschulinstitut für internationale Studien (HEI) tätig, hatte einen illustren Kreis eingeladen, um ein Thema zu diskutieren, das seitdem nicht an Aktualität verloren hat, weil es Grundlagen der sich ändernden Völkerrechtsordnung betrifft.

Das herkömmliche Völkerrecht baut auf Gegenseitigkeitsbeziehungen zwischen den Staaten auf: Der Staat ist nicht nur an der Entstehung ihn bindender Völkerrechtsnormen beteiligt, er muss seine Rechte auch gegenüber anderen Staaten durchsetzen. Die These der Herausgeberin lautet nun, dass mit der Entwicklung fundamentaler Gemeinschaftsnormen (wie sie im ius cogens ihren Ausdruck finden) und ebensolcher Gemeinschaftsinteressen auch das System der Rechtsdurchsetzung Schritt halten müsse. Gebraucht würden zentrale Mechanismen. So kommen ins Blickfeld das Kapitel VII der UN-Charta und der Sicherheitsrat, dem die Hauptverantwortung für die Wahrung des Weltfriedens übertragen ist und der Sanktionen gegenüber Friedensstörern verhängen kann. Sanktionen dienen, wie AbiSaab in seiner keynote address feststellt, der Absicherung der normativen Integrität des Rechtssystems und damit auch, wenngleich nicht notwendig, der Rechtsdurchsetzung.

Die einzelnen Beiträge beschäftigen sich in drei großen Themenblöcken mit diesen Sanktionen. Am Anfang stehen theoretische Grundfragen, die wiederum untergliedert werden in mehrere Einzelthemen. Die Einordnung der Sanktionen in das Völkerrechtssystem behandeln Pierre-Marie Dupuy (Entwicklung der Sanktionspraxis des Sicherheitsrats) und James Crawford (Verhältnis zwischen Sanktionen und Gegenmaßnahmen). Der rechtlichen Einbindung des Sicherheitsrats sind zwei weitere Referate gewidmet, wobei die Untersuchung der gerichtlichen Kontrolle des Sicherheitsrats John Dugard Gelegenheit gibt, sich mit den Fällen Lockerbie (Provisional Measures) und Application of the Genocide Convention (Provisional Measures) auseinander zu setzen. Nach Alternativen zu kollektiven Wirtschaftssanktionen fragen Andreas Lowenfeld, der sich dem amerikanischen Unilateralismus zuwendet (wobei er den Helms-Burton Act für völkerrechtswidrig hält), Alain Pellet, der auf die individuelle strafrechtliche Verantwortung als Mittel zur Rechtsdurchsetzung 
eingeht, und Bruno Simma mit seinen Bemerkungen zur Rechtsdurchsetzung durch Regionalorganisationen, wobei er kritisch auf den Kosovo-Einsatz der NATO zu sprechen kommt.

Im zweiten Teil werden die humanitären Implikationen von Sanktionen behandelt. Dabei geht es um die Achtung der Menschenrechte, etwa des Rechts auf Nahrung, wie auch der Genfer Konventionen bei der Verhängung und Durchführung von Sanktionen. Erfreulicherweise kommen hierzu auch die Praktiker von UNICEF, ILO und IKRK zu Wort.

Der dritte Hauptteil wendet sich den Auswirkungen von UN-Sanktionen auf das Privatrecht und weiteren Spezialproblemen zu. Ausführlich behandelt Geneviève Burdeau das Schicksal privatrechtlicher Verträge im Fall von Wirtschaftssanktionen, während Jochen A. Frowein über die Umsetzung in Deutschland schreibt, was Eingriffe in privatrechtliche Handelsverträge auf der Grundlage des EG-Außenhandelsrechts und des deutschen Kriegswaffenkontrollgesetzes einschließt. Die Sonderthemen gehen ein auf die Europäische Union, die Durchsetzung eines Wirtschaftsembargos auf See, die Stellung der von Sanktionen negativ betroffenen Drittstaaten gemäß Art. 50 UN-Charta, die Sanktionspraxis in Afrika und - inzwischen überholt - auf Nichtmitglieder der Vereinten Nationen am Beispiel der Schweiz.

Ein kurzer Ausblick (,The Future of Sanctions”) oszilliert zwischen einer Hinwendung zu mehr Prävention (Christine Chinkin), Abschaffung von Wirtschaftssanktionen (so der frühere irakische Botschafter Amir Al-Anbari) und mehr Demokratie durch die Beseitigung des Vetos (Monique Chemillier-Gendreau) - nicht gerade wirklichkeitsnahe Vorschläge. Damit schließt ein Buch, das eine Vielzahl von Themen im Zusammenhang mit UN-Sanktionen aufgreift, sie aber reichlich disparat behandelt. Ein guter Problemaufriss und Anregung zum Weiterdenken, nicht mehr!

Ulrich Fastenrath, Dresden

\section{Frank Semper}

\section{Die Rechte der indigenen Völker in Kolumbien}

SEBRA-Verlag; Hamburg, 2003, 382 S., € 55,00

Der Verfasser ist als erfahrener Kolumbien-Kenner ausgewiesen durch einen spannenden Reisebericht (Tor zum Amazonas, 1999) sowie den bewährten, zusammen mit Hella Braune herausgegebenen Reiseführer (Kolumbien Reisekompass, 2. Aufl. 2001). Nunmehr widmet sich der gelernte Jurist in seiner Frankfurter Dissertation den rechtlichen und faktischen Problemen der kolumbianischen Indianerschutzpolitik, eine Themenstellung, die in dieser Form nur aufgrund seiner engen Vertrautheit mit dem Land sinnvoll bearbeitet werden konnte. Zur Illustration dient eine bebilderte Einlage, in der er über seine wiederholten Besuche bei verschiedenen kolumbianischen Indianergemeinschaften berichtet. 
Heute leben noch ca. 600.000 Indianer in Kolumbien, die weniger als $2 \%$ der Gesamtbevölkerung ausmachen, denen aber die offizielle Gesetzgebung ca. $25 \%$ der staatlichen Landfläche zuspricht. Eine eingehende Darstellung der vielfältigen - über 80 - indianischen Ethnien und ihrer ganz unterschiedlichen sozio-kulturellen Strukturen bildet die Grundlage der folgenden Untersuchung. Zuvor stellt der Verfasser sein Thema einleitend in den geschichtlichen und internationalen Zusammenhang und versucht auf diesem Hintergrund die grundlegenden Begriffe zu klären. Die neuere kolumbianische Gesetzgebung knüpft nicht wie andere Rechtsordnungen an den Begriff des „Indio“ oder „Indígena“ als persönlichen Statuts an, sondern wählt die ,,indigene Gemeinschaft“ zum Ausgangspunkt. Demgegenüber hält der Verfasser den Begriff der ,indigenen Völker“ für eher geeignet, der vielschichtigen Realität gerecht zu werden, auch wenn die völkerrechtlichen Implikationen dieser Terminologie noch nicht befriedigend geklärt erscheinen.

Der Hauptteil der Arbeit enthält eine detaillierte Übersicht über die Indigenen-Gesetzgebung seit der Unabhängigkeit Kolumbiens bis zur geltenden Verfassung von 1991 sowie eine eingehende Analyse der einschlägigen Verfassungsbestimmungen. Die ältere Gesetzgebung bestand nach der Feststellung des Verfassers aus einem Konglomerat widersprüchlicher Regelungen, die einerseits nach dem kolonialen Modell einer Sondergesetzgebung für die Indigenen gestaltet, anderseits auf deren Integration in die nationale Gesellschaft angelegt waren. Mit der Verfassung von 1991 wurde dagegen ein einheitlicher rechtlicher Rahmen geschaffen, der die Rechte der indigenen Völker in ihren unterschiedlichen Aspekten umfasst und deren konkrete Postulate sich zu einer authentischen „Indigenenverfassung" zusammenfügen (im Anhang in spanischer und deutscher Sprache abgedruckt). Ausgehend von der Grundnorm des Art. 7, der die ethnische und kulturelle Vielfalt der kolumbianischen Nation betont, erörtert der Verfasser die einzelnen territorialen, administrativen, sozialen und finanziellen Gewährleistungen der indigenen Rechte in der Verfassung, ihre gesetzliche Konkretisierung und ihre Auslegung durch die Rechtsprechung des Verfassungsgerichtshofs (zu dessen Bedeutung im politischen Spektrum Kolumbiens siehe Springer, VRÜ 2003, 324 ff.) in über 30 Leitentscheidungen. Die inhaltlich reiche und gründlich recherchierte Darstellung leidet etwas unter der unübersichtlichen Dezimalgliederung mit ihren bis zu sieben Untergliederungen. Von besonderem Interesse ist dabei der Abschnitt über die indigene Sondergerichtsbarkeit, die in Art. 246 der Verfassung ausdrücklich anerkannt wird und deren Verhältnis zum allgemeinen Rechtssystem zahlreiche methodische und praktische Fragen aufwirft (siehe dazu auch die neuere Entscheidung des Verfassungsgerichtshofs vom 25.10.2001, T-1127, Foro Colombiano 66, 203). Die eindrucksvolle Präsentation der kolumbianischen Indigenenverfassung kontrastiert allerdings mit der wiederholten Feststellung des Verfassers, dass die Praxis vielfach hinter ihren Forderungen und den dazu ergangenen gesetzlichen Regelungen zurückbleibt.

Diese Diskrepanz zwischen dem rechtlichen Schutzstandard und der praktischen Durchsetzung kennzeichnet auch den abschließenden Teil der Untersuchung, der das kolumbianische Indigenenrecht unter dem kritischen Aspekt der internationalen Menschenrechtskonventionen überprüft. Im Vordergrund stehen dabei der Pakt über bürgerliche und politische 
Rechte (1966), der Pakt über wirtschaftliche, soziale und kulturelle Rechte (1966), das Übereinkommen zur Beseitigung der Rassendiskriminierung (1966), die Völkermordkonvention (1948) sowie die Konvention Nr. 169 der Internationalen Arbeitsorganisation zum Schutz der indigenen Völker (1989). Jeder dieser internationalen Verträge, die alle von Kolumbien ratifiziert sind und damit dem einfachen Gesetzesrecht vorgehen (Art. 93 Verf.), wird vom Verfasser eingehend hinsichtlich seiner rechtlichen Garantien zugunsten der Indigenen, seiner Schutzmechanismen und seiner tatsächlichen Bedeutung untersucht. Im deutlichen Kontrast zu den rechtlichen Standards der Konventionen sieht er dabei die Situation in Kolumbien ,geprägt von der schweren und systematischen Verletzung elementarer Menschenrechtsnormen“, wie er dies detailliert für den Bereich der indigenen Völker belegt. Die zum Schutz der Menschenrechte geschaffenen staatlichen Institutionen in Kolumbien erweisen sich demgegenüber in ihrer derzeitigen Form als unzureichend. An diesem Punkt berührt sich die Arbeit mit einer gerade erschienenen Untersuchung, die für den mittelamerikanischen Raum zu ähnlichen Ergebnissen gelangt (Christine Binder, Die Landrechte indigener Völker unter besonderer Bezugnahme auf Mexiko und Nicaragua, 2004). Was die Situation in Kolumbien als besonders ausweglos erscheinen lässt, ist die vom kolumbianischen Gesetzgeber selbst anerkannte - Bürgerkriegssituation, in der die indigenen Völker zwischen die Fronten der kämpfenden Parteien geraten.

Mit seinem Buch hat Frank Semper eine umfassende und kompetente Darstellung des kolumbianischen Indigenenschutzrechts vorgelegt. Die Wirksamkeit des von ihm beschriebenen Rechtsinstrumentariums beurteilt er selbst eher skeptisch. Man spürt aber das unbedingte Engagement des Autors, dem die konkrete Verbesserung dieser Situation ein wesentliches Anliegen ist.

Jürgen Samtleben, Hamburg 


\section{Peter Hanser / Trutz von Trotha}

\section{Ordnungsformen der Gewalt}

Reflexionen über die Grenzen von Recht und Staat an einem einsamen Ort

in Papua-Neuguinea

Siegener Beiträge zur Soziologie, Bd. 3

Rüdiger Köppe Verlag, Köln, 2002, 448 S., € 49,80

Die Monographie hat zum Ziel, einen Beitrag zum Verständnis der Probleme der Gewährleistung von Recht und Ordnung in dem südpazifischen Inselstaat Papua-Neuguinea zu leisten. Es geht um die Bedingungen, Arbeitsweisen und Wirkungen der staatlichen Rechtsordnung an der Nahtstelle sich auflösender zentralstaatlicher Durchsetzungsfähigkeit und Kontrolle - der peripheren Grenze des (modernen) postkolonialen Staates - und lokaler Bedingungen der Autonomie, unter denen autochthone Formen der Streitregelung wirksam sind. Regionaler Forschungsschwerpunkt ist der Bezirk Ihu in der Gulf-Provinz, die westlich an die Central-Provinz mit dem Hauptstadtdistrikt angrenzt. Autoren sind der Ethnologe Peter Hanser, der im Jahr 1985 ein Buch zu den Ursachen kollektiver Gewaltanwendung in den Stammesgesellschaften Neuguineas veröffentlicht hat, und der in Siegen lehrende Soziologe Trutz von Trotha, der sich mit den umfassenden Themen Staat, Recht und Gewalt befasst. Hanser schloss seine 14-monatige Feldforschung vor Ort im April $1993 \mathrm{ab}$.

Die Studie ist in vier Teile gegliedert, wobei Einleitung und II. Teil von beiden Autoren verantwortet werden, Teil I und III hingegen nur durch von Trotha. Die Einleitung präsentiert ethnographische Grundlagen der Region. Das dünnbesiedelte Gebiet wird als staatlich vernachlässigt und wirtschaftlich stagnierend mit fast vorkapitalistischen Lebensverhältnissen charakterisiert. Trotzdem unterliegt es aufgrund von Bildung, dem (kaum nachhaltigen) Wirken von Religionsgemeinschaften und der Migration dem sozialen und kulturellen Wandel. Die zu der Sprachgruppe der Elema zählenden, patrilinear organisierten Verwandtschaftsgruppen werden von den Autoren als relativ friedlich ohne tiefgreifende politische Konflikte und endemische Kriegstendenzen vergleichbar dem Hochland bezeichnet. Dies manifestiert sich in einer Streitregelungskultur, in der zwar häufig mit tödlicher Gewalt gedroht, diese aber abgesehen von dem allgegenwärtigen Todeszauber selten realisiert wird. Abwarten und Zeitgewinnen wirken hier als konfliktentschärfendes Verhalten. Ein abschließender Exkurs thematisiert die Unsicherheitserfahrung im Land und das Problem der Bandenkriminalität (in Tok Pisin `raskolism`), beide Faktoren eher allgegenwärtig als nur auf die wenigen Städte beschränkt.

Teil I formuliert eine „Kritik der Rechtsethnologie“ hinsichtlich der Frage nach Inhalt und Anwendbarkeit einer Kategorie des Rechts, die sich seit Malinowski abweichend vom westlichen, von Juristen geprägten Verständnis auf den Grundsatz der Reziprozität mit seinen integrierenden Ansprüchen und Verpflichtungen bezieht. Gefragt wird nach einem Rechtsbegriff, der über westliche Gesellschaften mit ihren hoch institutionalisierten Rechtsinstanzen hinaus in der interkulturell vergleichenden Forschung Anwendung finden 
könnte. Ein Kapitel skizziert die Hinwendung der Ethnologie zur Streitregelung, die vergleichbar der These vom Rechtspluralismus, der nach von Trotha gegenwärtig einflussreichsten Sichtweise in der Rechtsanthropologie, kaum den Vorgängen in den Gesellschaften gerecht werde. Von Trotha zielt darauf, die besondere Qualität und Legitimität der Vorherrschaft staatlichen Rechts und seiner Einrichtungen in der Formulierung eines Rechtskonzepts zu berücksichtigen.

Ein Exkurs beantwortet die Frage, was Recht ist, mit der Herausbildung und Institutionalisierung des neutralen Dritten als gesellschaftlicher Vereinbarung bei der Streitregelung. Während bei reinen (rechtsfreien, nicht rechtlosen) Selbsthilfeordnungen deren Handhabe den autonomen Konfliktbeteiligten oder der Anrufung des Übernatürlichen obliegt, die sozialen Gruppen also die exklusive Verfügungsgewalt über die normative Ordnung, die Voraussetzung jeglicher Vergesellschaftung, inne haben, wird ihnen diese mit der Herausbildung einer Überparteilichkeit und Allgemeingültigkeit beanspruchenden Rechtsordnung entwunden. Sie repräsentiert die Gesellschaft als Ganzes und deren allgemeines Interesse an der Aufrechterhaltung der Ordnung, auch wenn die vollzogene Entmachtung der Akteure durch die monopolisierte staatliche Rechtsordnung nie einhellig ist. Der Autor skizziert dann typologisch die Institutionalisierung des Rechts von der kommunitären Rechtsordnung über Häuptlingstümer und Großhäuptlingstum (nichtstaatliche Ordnungen) hin zur staatlichen Rechtsordnung, die sich jeweils nach Grad und Zentralisierung politischer Herrschaft unterscheiden. Der Schrecken der gewaltsamen Selbsthilfe, der Preis der politischen Freiheit egalitärer Verwandtschaftsgruppen, weicht der Angst vor den willkürlich und despotisch Herrschenden, bis die Gewalt schließlich rechtsstaatlich eingehegt ist. Dies aber stellt weltgeschichtlich eher die Ausnahme als die Regel dar.

Der zweite Teil zeigt detailliert die Realität und Begrenztheit staatlicher Einheit und Rechtsordnung in der Gulf-Provinz auf, wobei schon der flächenmäßig größte Teil des IhuBezirks landeinwärts offiziell als non-governmental area eingestuft ist, also außerhalb dauerhaften staatlichen Einflusses liegt. Die Arbeit der Strafverfolgungsbehörden ist von Durchsetzungsunfähigkeit und Ohnmacht aber auch von Verharmlosung gekennzeichnet. Schwere Vergehen wie Raubüberfälle, Ritualmorde (Zauberei) und Sexualdelikte an Minderjährigen bleiben ungesühnt, da in den ländlichen Regionen weder Täter noch Zeugen greifbar sind. Ein weiteres Kapitel widmet sich der Arbeitsweise des Dorfgerichts, das die Brücke zwischen kommunitärer Selbsthilfeordnung und Gerichtssystem mit District, National und Supreme Court (nicht Local Court) darstellen soll, allerdings auch den Verfassungsprinzipien unterliegt und damit Teil der Rechtsinstanzen ist. Der Zirkel der gewaltsamen Selbsthilfe wird letztlich damit aber nicht zerbrochen. Stattdessen, so die Schlussfolgerung Hansers und von Trothas, wird ihm eine weitere Kette gerichtlicher Streitforen hinzugefügt. die zudem durch den Kampf um möglichst hohe monetäre Kompensation belastet ist. Ein letztes Kapitel zeigt die Konflikte um Macht, Status und Einfluss der von big-men geprägten Verwandtschaftsgruppen. Die im Local Government Council (seit 1995 LocalLevel Government) engagierten Führer bestehen auf ihrer kolonialen Erfahrung der Einheit 
von Politik und Recht, um die mit ihnen konkurrierende neuere Dorfgerichtsbarkeit zu diskreditieren.

Die Zukunft des staatlichen Gewaltmonopols wird im dritten Teil thematisiert. Von Trotha präsentiert eine Typologie gesamtgesellschaftlicher Ordnungsformen der Gewalt, die auf Studien über Westafrika (der bäuerlich-familiär geprägten neo-despotischen Ordnung), Lateinamerika (einer von Urbanität und Verelendung gekennzeichneten Ordnung der vervielfältigten Gewalt) und Europa (wohlfahrtsstaatliche Ordnung) beruht. Die westlichen Demokratien sieht der Autor bereits durch einen Untergang des Staates und den Rückgang der hohen Sicherheitsleistung in Folge einer Privatisierung bedroht, was die einzigartige Entwicklung von domestiziertem Gewaltmonopol und Rechtsstaat aushöhlen und damit die „Erste“ der „Dritten Welt“ näher bringen könnte. Papua-Neuguinea wird als eigene Ordnung der gewalttätigen Verhandlung und staatlichen Untätigkeit eingestuft. Das Land teile zwar viele Erscheinungsformen mit der neo-despotischen Ordnung, unterscheide sich aber durch die soziale Egalität und das fehlende Häuptlingswesen sowie das parlamentarische System und die Gewaltenteilung. Weiter wachsende Bandenkriminalität, Stammeskämpfe und Landkonflikte deuten darauf hin, dass Gewalt sowohl veralltäglicht als auch durch den schwachen Staat eingegrenzt bleibt, die gewalttätige Selbsthilfe allerdings Zulauf erhält.

Der abschließende Eindruck des anspruchsvollen Werkes bleibt ambivalent, was sich insbesondere an der Komposition des Bandes festmachen lässt. Einerseits ermöglicht die Mikroanalyse provinzialer Wirklichkeit einen tief greifenden Einblick in die Wirkungszusammenhänge und Grenzen moderner, schwach ausgeprägter Staatlichkeit versus sich behauptender tradierter Lebenszusammenhänge, eine Zustandsbeschreibung, die sich in vielen vernachlässigten Regionen Papua-Neuguineas, aber auch in den Provinzen mit wirtschaftlichen Wachstumspolen beobachten lässt. Es ist ein wichtiger Baustein zum Verständnis der komplexen und widersprüchlichen, historisch wie aktuell bedingten gesellschaftlichen Zustände und Entwicklungen, die gerade in den letzten Jahren den sich beschleunigenden Zerfall zentralstaatlicher Strukturen nahe legen. Dieser Aspekt fand allerdings wie auch die neuere Literatur trotz der späten Publikation fast zehn Jahre nach Abschluss der Feldforschung keine Berücksichtigung.

Andererseits handelt es sich bei dem ersten und dritten Teil um überarbeitete Versionen von Veröffentlichungen von Trothas der Jahre 1987, 2000 und 1995. Statt des Wiederabdrucks der Einwände gegenüber der kritischen mainstream-Rechtsethnologie der 1970er Jahre, etwa Stanley Diamond und Uwe Wesel, hier charakterisiert als „Romantiker der kommunitären ,Wildheit'“ (S. 149), hätte man sich einen Überblick über die Fortentwicklung der Rechtsanthropologie in den vergangenen 20 Jahre gewünscht. Auch kommt das Bild, das von Papua-Neuguinea gezeichnet wird, mit jeweils wenigen Seiten viel zu kurz. Dies insbesondere hinsichtlich des in Teil III angekündigten Blickes auf das politische Ganze des Landes, das die Hinterlandperspektive mit der Typologie von Gewaltformen verbinden soll.

Mehr noch versucht man manchmal vergeblich, das Land in dem Beschriebenen wieder zu finden. Dies beispielsweise, wenn von Elendsvierteln und Migration bei der Kriminalitäts- 
entwicklung Anfang der 1970er Jahre die Rede ist (das unzureichende Kapitel zum Bandenwesen in der Einleitung wird zudem plötzlich unterbrochen und mit einer Beschreibung der Situation der christlichen Kirchen fortgesetzt) oder die Durchsetzung kolonialer Herrschaft beschrieben wird. Die Vielschichtigkeit und zeitliche Perspektive der Entwicklungen erscheint kaum angemessen wiedergegeben. Erfahrungen aus Afrika waren hier möglicherweise leitender als die Gegebenheiten und die spezifische koloniale Geschichte des Südpazifikstaates. Auch wären Fehler vermeidbar gewesen. Weder stimmt die behauptete koloniale britisch-deutsche Grenzziehung entlang des achten südlichen Breitengrades (zumindest für den Großteil des Festlandes bis zum 147. östlichen Längengrad), noch gab es die nennenswerte antikoloniale Bewegung oder den Handel mit Haschisch. Die Kritik soll die vielen zutreffenden über Papua-Neuguinea hinaus gehenden Erkenntnisse des Buches nicht schmälern. Es ist zudem eine wertvolle Grundlage und Motivation für weitere Forschungen zur Konstituierung postkolonialer Staatlichkeit.

Roland Seib, Darmstadt

Werner Draguhn (Hrsg.)

Indien 2003

Institut für Asienkunde, Hamburg, 2003, 482 S., € 30,00

Seit 1998 erscheint dies Jahrbuch kontinuierlich zum 6. Mal. Der Herausgeber kann sich auf eine Gruppe ständiger Autoren verlassen: Von den 16 dieser Ausgabe hat die Mehrheit mindestens bereits in 4 früheren Jahrbüchern an den Themen Innen- und Außenpolitik, Wirtschaft und Gesellschaft sachverständig mitgewirkt.

J. Betz (S. 31 ff.) berichtet über die Wahl des neuen Staatspräsidenten Abdul Kamal, angesehener Architekt des indischen Raketenprogramms und kein Politiker. Der bisherige Präsident Narayanan hatte mehrfach gegen den hinduistischen Kurs der Regierung Vajpayee opponiert. Die oppositionelle Kongresspartei aber hatte ebenso wenig wie die Regierungskoalition eine Mehrheit im electoral college (Bundesober- und -unterhaus) sowie die Landesparlamente, Art. 54 und 55 der Verfassung). Ebenso behandelt Betz die Auseinandersetzungen zwischen Hindus und Moslems im westlichen Bundesstaat Gujarat und die dann im Dezember 2002 von der in diesem Staat regierenden und für den Mord an Hunderten Moslems mitverantwortlichen Partei BJP haushoch gewonnene Landtagswahl.

Auf diese Wahl geht $H$. Reifeld in seinem Beitrag mit dem Titel „Gujarat 2002: Menetekel für Indien?" im einzelnen ein (S. 53 ff.).

D. Rothermund (S. 43 ff.) führt auf die Entstehung des Kashmir-Problems im Jahre 1947 zurück. Lord Montbatten, noch Generalgouverneur von Indien, bekam vom pakistanischen Regierungschef Jinnah gesagt, mit dem Einsatz regulärer pakistanischer Truppen in Kashmir nichts zu tun zu haben. Diese heuchlerische Position hat die pakistanische Regierung 
bis in die Gegenwart beibehalten. Erst neuerdings scheint General Musharraf eine realistischere Darstellung zu gestatten. Die Lage im Kashmir hat sich seit den Wahlen 2002 auch durch die neue Koalitionsregierung von Kongresspartei und People's Democratic Party (PDP) unter Mufti Mohammed Sayeed deutlich entspannt.

Schwierig sieht es auch im Nordosten Indiens aus, wo sich in dem in 6 Gliedstaaten aufgeteilten Assam mit Massakern und Entführungen Separatisten durchzusetzen versuchen (T. Schmitt, Assam und die Bodoland-Bewegung, S. 79 ff.).

J.A. Dohrmann berichtet (S. 93 ff.) über die Lok Adalats, eine Art Volksgerichte, welche die ordentlichen Gerichte um kleinere Fälle entlasten und der Bevölkerung einfachere und formfreiere Streitschlichtung ermöglichen sollen, und über die Geschichte der Wirksamkeit des hoch angesehenen Supreme Court vor, während und nach den Notstandsjahren 1975 1977 und über dessen die Gewaltenteilung verwischende Ausdehnung seiner Rechtsprechung in legislative und exekutive Bereiche.

In Elena Koch's Papier über Medien in Indien (S. 117 ff.) findet sich der Hinweis, dass das staatliche All India Radio (AIR) im Inland in 24 Sprachen und 146 Dialekten sendet, der Auslandsdienst in 16 nationalen und 10 ausländischen Sprachen. Für die 120 Mio. Radios (im Jahr 2000) hat AIR inzwischen zwar UKW-Frequenzen - zu hohen Lizenzgebühren für private Anbieter freigegeben. Diese dürfen jedoch keine Nachrichten senden. N.-C. Schneider ergänzt mit Ausführungen ,Zum Machtfaktor ,Presse' in Indien“ (S. 143).

Für an den indischen Verfassungsprinzipien ausgerichtetes und die Folgen falscher Erziehung beachtendes Denken sind die Zitate von M. Gottlob (Arbeit am Feindbild: Der Streit über den Geschichtsunterricht an Indiens Schulen, S. 171 ff.) aus neuen Schulbüchern der politischen Richtung Sangh Parivar/RSS/BJP erschreckend - wenn z.B. Muslime, Christen und Parsen als Ausländer etikettiert werden und Mahatma Gandhis Ermordung (durch RSS-Kreise!) nicht einmal erwähnt ist.

C. Wagner (S. 205) untersucht Indiens Machtstellung im Staatengefüge und nennt es ,eher eine Großmacht im Wartestand (...), denn Armut und Unterentwicklung gehen kaum mit der Vorstellung einer Großmacht zusammen.“

K. Frey (S. 221 ff.) sieht Möglichkeiten zur Stabilisierung der nuklearen Konfrontation im Ausbau der Führungs- und Kontrollstrukturen, der Kommunikation und Aufklärung sowie der Zweitschlagsfähigkeit. Bei H. Kreft (Die USA zwischen Indien und Pakistan, S. 239 ff.) lautet ein Zwischentitel: „US-amerikanischer Pragmatismus: India first bei gleichzeitigem Festhalten an Pakistan“.

Abgesehen von der großen, weit verbreiteten Armut von gut einem Viertel des Milliardenvolkes kann Indien mit ansehnlichen wirtschaftlichen Wachstumsraten aufwarten (J. Mertens / S. Deo, Die indische Wirtschaft im Berichtsjahr, S. 259 ff.). Es hat in den Jahren 2001/02 und 2002/03 seit über zwei Jahrzehnten wieder Liquiditätsüberschüsse erzielt (1,35 bzw. 1,67 Mrd. US\$). Die Devisenreserven betragen 82 Mrd. US\$, das Haushaltsdefizit allerdings 5,5 \% des BSP. Der Bundeshaushalt 2003/04 ist auf $€ 86$ Mrd. geplant mit Akzent auf Investitionen in die Infrastruktur.) Zu Entwicklung und Perspektiven der indischen Außenhandelsstruktur liefern M. von Hauff und N.V. Michaelis (S. 271 ff.) 
Angaben über Indiens geringen Anteil am Welthandel, sein hohes Handelsdefizit und zu der sich vom primären zum sekundären Sektor verlagernden Struktur des Außenhandels. Laut S. Gosalia (S. 287 ff.) hat sich der Dienstleistungssektor (54\% des BSP) am schnellsten entwickelt gegenüber Industrie (23\%) und Landwirtschaft $(27 \%)$. Letztere jedoch noch mit über $50 \%$ der Beschäftigten.

$D$. Bronger und J. Wamser bringen die dritte Fortsetzung ihres Vergleichs Indiens mit China mit umfangreichen Wirtschaftszahlen über beide Länder (S. 305 ff.). W.-P. Zingel (S. 353 ff.) stellt fest, dass die Agrarproduktion weit über das Bevölkerungswachstum hinaus gesteigert und verbessert werden konnte, auch die Zahl der absolut Armen zurück ging, aber noch nicht erreicht wurde, dass alle Inder ausreichend zu essen haben.

M. Haack (S. 379 ff.) diskutiert die, entsprechende Schwierigkeiten europäischer Großstädte weit übersteigenden Schwierigkeiten der indischen Großstädte, von denen 35 zwischen 16 Mio. (Bombay) und 1 Mio. (Rajkot in Gujarat) Einwohner zählen. Wie überhaupt bei vielen der in den Darstellungen dieses Jahrbuchs behandelten Themen sich z.B. nationale deutsche Probleme wie Unterhaltungspossen im Verhältnis zu wirklichen Problemen ausnehmen. Wünschenswert wäre im nächsten Jahrbuch sicherlich eine Analyse der Wasserproblematik in Indien.

Den Abschluss des hervorragend informativen und nützlichen Bandes bilden ein Überblick über Unternehmensbesteuerung in Indien (R. Schmid / C. Kruchten, S. 395 ff.) sowie Chroniken politischer und wirtschaftlicher Ereignisse (M. Schied, S. 413 ff., 423 ff., 431 ff.), eine Kurzbiographie zu Gesellschaftsstruktur und sozialen Bedingungen in Indien $(S$. Majumder, S. 441 ff.) sowie ein wirtschaftsstatistischer Anhang (S. 465 ff.).

A. Albano-Müller, Schwelm

\section{Yongping Ge}

\section{Hongkong und die Europäische Union}

Auch ein Beitrag zur Völkerrechtssubjektivität und Vertragsabschlußkompetenz

Wissenschaft in Dissertationen, Band 726

Görich \& Weiershäuser Verlag, Marburg, 2003, xiv, 293 pp, paper

In any good whodunnit, the identity of the murderer is revealed as a dramatic conclusion. In a good scholarly dissertation, the issue prompting the enquiry is mostly stated at the outset, and the author then takes the reader step by step towards answers to problems identified at the beginning. Dr Ge's doctoral thesis on Hong Kong and the European Union (EU), and in particular the questions of their legal personality and treaty-making capacity, leaves the reader in suspense until the end as to the objective of his presentation: Is it an attempt to establish whether lack of legal personality or treaty-making capacity in any one of the entities discussed have been or may be an obstacle to mutual relations? It turns out 
that no specific question thematically linking the Special Administrative Region (SAR) of Hong Kong and the EU has been defined as the point of departure for this monograph but that the author has chosen to juxtapose the two in abstract comparison. Such abstract investigation may still be a worthwhile labour even though it lacks the analytical perspective derived from a guiding issue.

In five extensive chapters, the author summarises the historical, legal and institutional foundations of the EU and Hong Kong; the scope of legal personality of the European Communities (EC), the EU and Hong Kong; the treaty-making capacities of the EC and Hong Kong; relations of the EC and the EU with Hong Kong; and legal personality and agreements in respect of the EC, the EU and Hong Kong.

The description of the historical and institutional backgrounds of the EC/EU and Hong Kong retreads thoroughly familiar ground. The lack of express legal personality - and corresponding treaty-making capacity - of the EU, as distinct from the EC, is rightly seen as never having impeded conclusion of the relevant agreements underpinning co-operation between the two sides, with the EC acting as the legal personification of the European party.

There thus appears to be little added value in the lengthy comparisons of the EC/EU and Hong Kong, quite apart from the fact that both are results of very different processes. The former represents the fruit of a voluntary political project, initiated in particular before the European backdrop of two 'world' wars, to coalesce the sovereign states of the Old Continent in a larger whole where their destructive rivalries of the past would be removed, or at least domesticated, and their productive potentials could be enhanced for the profit of their citizens and to the benefit of such an enlarged group's standing on the international stage. The latter, rather than an active participant in an integrative process, has been the legally voiceless object of a diplomatic compromise in 1984 between the United Kingdom, whose long-term grip on this Crown Colony - to be mortally truncated by the expiry of the lease on the New Territories - had become tenuous to the point of being unable to survive even a suspension of water and vegetable supplies from the mainland, and an irredentist Chinese government adamant in its determination to wipe from the map the last traces of the "First Opium War" (1840-42). Besides, since the establishment of the SAR in 1997, Hong Kong's terms of trade vis-à-vis the mainland have eroded in the face of rapid development in growth centres of China, such as Shanghai and the neighbouring Pearl River Delta. Foreign investors who may previously have preferred to procure banking or quality control services of mainland manufacturing operations from Hong Kong, where they often also chose to locate their regional presence, can now reliably do this at lower cost in China proper. The new economic embrace of the SAR in the "Closer Economic Partnership Arrangement between Hong Kong and the Chinese mainland" (CEPA) testifies not only to the economic logic flowing from Hong Kong's proximity to and numberless links with the interior of the People's Republic. It also points to a vulnerability of the territory that may no longer unquestioningly count itself as the golden goose which its new Peking owner would be prepared to handle gingerly in expectation of more eggs to come. In the European 
Union, expansion of membership and reform of its institutions in the new constitution would also have brought the EU legal personality, but the setback at the end of last year in adopting the new instrument has delayed this addition to the EU's juridical panoply. Meanwhile both China and the EU have been expanding their partnership in numerous fields, unaffected by differences of their legal make-up. Dr Ge's occasionally instructive dissertation makes for a bland whodunnit, without a corpse and without a denouement.

Wolfgang Kessler, Peking ${ }^{*}$

The author is Head of the Cultural Section of the Embassy of the Federal Republic of Germany in Peking; the above review expresses his own personal views. 